\title{
The role of ITO resistivity on current spreading and leakage in InGaN/GaN light emitting diodes
}

\author{
V. Sheremet ${ }^{a}{ }^{*}$, M. Genç ${ }^{b}$, M. Elçi ${ }^{a}$, N. Sheremet ${ }^{c}$, A. Aydınlı ${ }^{\mathrm{a}, \mathrm{d}}$, I. Altuntaş ${ }^{\mathrm{e}}$, \\ K. Ding ${ }^{f}$, V. Avrutin ${ }^{f}$, Ü. Özgür ${ }^{f}, H$. Morkoç ${ }^{f}$ \\ a Advanced Research Laboratories, Department of Physics, Bilkent University, Ankara, 06800 Turkey \\ b Optoelectronic R\&D Center, Ermaksan, Bursa, 16065, Turkey \\ ${ }^{\mathrm{c}}$ Institute of Physics, National Academy of Sciences of Ukraine, Kyiv, 03680, Ukraine \\ d Department of Electrical and Electronics Engineering, Uludağ University, Bursa, 16059, Turkey \\ e Department of Nanotechnology Engineering, Cumhuriyet University, Sivas, 58140, Turkey \\ ${ }^{\mathrm{f}}$ Department of Electrical and Computer Engineering, School of Engineering, Virginia Commonwealth University, Richmond, VA, 23284- \\ 3068, USA
}

\section{A R T I C L E I N F O}

\section{Article history:}

Received 15 June 2017

Received in revised form 6 August 2017

Accepted 8 August 2017

Available online 9 August 2017

\section{Keywords:}

InGaN/GaN multiple quantum well

LED

Indium Tin Oxide

Current spreading

\begin{abstract}
A B S T R A C T
The effect of a transparent ITO current spreading layer on electrical and light output properties of blue InGaN/GaN light emitting diodes (LEDs) is discussed. When finite conductivity of ITO is taken into account, unlike in previous models, the topology of LED die and contacts are shown to significantly affect current spreading and light output characteristics in top emitting devices. We propose an approach for calculating the current transfer length describing current spreading. We show that an inter-digitated electrode configuration with distance between the contact pad and the edge of $\mathrm{p}$-n junction equal to transfer length in the current spreading ITO layer allows one to increase the optical area of LED chip, as compared to the physical area of the die, light output power, and therefore, the LED efficiency for a given current density. A detailed study of unpassivated LEDs also shows that current transfer lengths longer than the distance between the contact pad and the edge of p-n junction leads to increasing surface leakage that can only be remedied with proper passivation.
\end{abstract}

(c) 2017 Elsevier Ltd. All rights reserved.

\section{Introduction}

Light emitting diodes (LEDs) based on InGaN/GaN semiconductor heterostructures have become the key components of solid-state lighting. Standard InGaN/GaN LED dies consist of multiple InGaN quantum well active regions sandwiched between highly doped n- and p-type gallium nitride [1]. Due to limited availability of bulk GaN substrates [2], vast majority of InGaN LEDs are grown on foreign substrates such as sapphire, which require extra efforts to reduce lattice mismatch induced defects that can cause nonradiative recombination and leakage currents. In addition, the use of nonconductive substrates, unless they are removed, dictates planar contact configuration which seriously impacts the current distribution due to low electrical conductivity of p-GaN [3,4]. Consequently, proper device designs and contact geometries must be considered to achieve effective current spreading.

\footnotetext{
* Corresponding author.

E-mail address: sheremet@bilkent.edu.tr (V. Sheremet).
} 
Despite significant progress in InGaN/GaN LED processing, poor current spreading caused largely by low carrier mobility ( $\leq 10 \mathrm{~cm}^{2} / \mathrm{V} \mathrm{s}$ [3]), low electrical activation of acceptors (typically $<10^{18} \mathrm{~cm}^{-3}$ ) [4], and the resulting high resistivity of p-GaN layers $(\sim 1 \Omega \cdot \mathrm{cm}[4])$ is a major challenge. Use of nontransparent contacts on p-GaN mitigates somewhat, but does not eliminate current crowding which causes preferential light generation mostly close to the contact pads. Using flip-chip configuration that facilitates light emission from the backside of LED wafer is one of the ways to solve this problem. When the flip-chip approach is not feasible, use of a current spreading layer on the top of p-GaN layer aids in spreading the current more effectively. Transparent conductive oxides, such as indium tin oxide (ITO), are used as current spreading layers (CSLs) [1], due to their high transparency in the visible range and high conductivity $[5,6]$.

Current spreading in LED dies has been studied theoretically by Guo and Shubert [7,8] and Thomson [9]. The proposed models allow estimation of current spreading in LEDs by calculating a current spreading length for current flow through the full stack of LED layers. This is a characteristic length defined as the distance from the edge of the contact to a position where the current decreases to 1/e of its maximum at the edge of the contact. According to the proposed models, current spreading length depends on the n-type and p-type layer resistivities and their thicknesses [7,8] as well as the current density [9]. As regards to InGaN LEDs, one drawback of these models is that contacts to p-type semiconductor layers are considered as equipotential surfaces and their resistivities are neglected [7-9]. However, ITO used for ohmic contacts to p-GaN layers can have a resistivity that is much larger than that of a typical metal. Hence, finite resistivity of ITO $\left(\sim 10^{-4} \Omega \cdot \mathrm{cm}^{2}\right)$ should be taken into account in the calculation of current spreading length [5].

Influence of the current spreading layer on the properties of InGaN/GaN based LED structures has also been explored by Kim et al. [10-14]. In these works, LED efficiency has been found to depend on the die size as well as the resistivity of the ptype GaN contact layer. It has also been proposed that, in order to avoid current crowding, it is necessary to fabricate LEDs with n-GaN and current spreading layers of equally resistivities [10-14]. In the light of this work, on limited current spreading in InGaN/GaN based LEDs, several avenues were explored to improve current spreading and efficient use of die area. They include interdigitated configuration contacts on LED dies [15-17] with current blocking layer under contact pads [15,16] and with various transparent conductive layers [17].

However, all these reports [12-23], used the models of Guo and Shubert [12,18,19] and Thomson [13-17,20-23], and therefore, did not take into account the limited conductivity of the ITO layer and the optical area of the LED die. To accurately calculate current densities, the active area of the die should be determined by taking into consideration the current transfer length in current spreading layer. We propose a model which uses a transfer length as a characteristic parameter for current spreading efficiency for structures involving current spreading layers. This parameter defines the optical area of the die that can contribute for light generation. Increasing the physical area of LED chip without taking into account the effects of current spreading on the die design would not necessarily improve the LED performance. Using LED die size as a parameter, we studied the current-voltage (I-V) and current-power (I-P) characteristics of blue LEDs and correlated them with the associated transfer lengths. In order to further understand the role of transfer length in current spreading, we used unpassivated interdigitated LED structures to study the relationship between the transfer length and leakage current (non radiative). We find that a proper design that takes into account the transfer length and passivation results in enhanced output light power.

\section{Experimental}

InGaN/GaN MQW based LED structures grown by metalorganic chemical vapor deposition (MOCVD) technique on sapphire substrates were used in this work. Epitaxial structures consist of $10-\mu \mathrm{m}$ thick undoped GaN buffer layers, followed by 4 $\mu \mathrm{m}$ thick $\mathrm{n}-\mathrm{GaN}\left(3 \times 10^{18} \mathrm{~cm}^{-3}\right), 60-\mathrm{nm}$ In $_{0.01} \mathrm{GaN}$ serving to reduce the probability of strain relaxation in the active region, 7 step graded $\operatorname{In}_{\mathrm{x}} \mathrm{Ga}_{1-\mathrm{x}} \mathrm{N}$ electron injector ( $\mathrm{x}=0.04$ to 0.1 with $3 \mathrm{~nm}$ thickness for each step), $6 \times 2 \mathrm{~nm} \mathrm{In}_{0.12} \mathrm{GaN}$ quantum wells separated by $3-\mathrm{nm}$ thick $\operatorname{In}_{0.06}$ GaN barriers, and $100-\mathrm{nm}$ p-GaN $\left(4 \times 10^{17} \mathrm{~cm}^{-3}\right)$. The step graded electron injector is included in the structure to help cool the electrons [24,25] and mitigate the electron overflow. Two LED designs were employed in this study. First one with square mesas of $300 \times 300 \mu \mathrm{m}^{2}$ (small), $600 \times 600 \mu \mathrm{m}^{2}$ (middle) and $1000 \times 1000 \mu \mathrm{m}^{2}($ large) in size and second one with interdigitated electrode patterns of $360 \times 360 \mu \mathrm{m}^{2}$ (small), $660 \times 660 \mu \mathrm{m}^{2}$ (middle) and $1060 \times 1060 \mu \mathrm{m}^{2}$ (large) in size. Mesas were formed by inductively coupled plasma (ICP) etching $\left(\mathrm{BCl}_{3}: \mathrm{Cl}_{2}\right)$. Ohmic contacts to $\mathrm{n}$-GaN were formed using a $\mathrm{Ti}(30 \mathrm{~nm}) / \mathrm{Al}(50 \mathrm{~nm}) / \mathrm{TiB}_{2}(50 \mathrm{~nm}) / \mathrm{Au}(70 \mathrm{~nm})$ stack which underwent rapid thermal annealing (RTA) in $\mathrm{N}_{2}$ at $\mathrm{T}=800{ }^{\circ} \mathrm{C}$ for $30 \mathrm{~s}$. Serving as current spreading layer and ohmic contact to p-GaN, $\mathrm{ITO}(130 \mathrm{~nm})$ or Ni(5 nm)/Au(5 nm)/ ITO(120 nm) were used with $\mathrm{Ni}(30 \mathrm{~nm}) / \mathrm{Au}(50 \mathrm{~nm})$ contact pads. ITO was deposited by RF sputtering in Ar ambient. Selected LED structures were passivated with $250-\mathrm{nm}$ thick $\mathrm{SiO}_{2} . \mathrm{SiO}_{2}$ was grown by the plasma enhanced chemical vapor deposition (PECVD) technique immediately after ICP mesa-etching. Lithography and subsequent etching in a buffered oxide etchant was used to open windows in $\mathrm{SiO}_{2}$.

Electrical properties of fabricated LED dies were measured using a Keithley 2430 source and measurement unit and a HP 4142B parameter analyzer. Optical properties were studied by Instrument Systems spectrometer CAS 140CT. Ohmic contact resistivities were determined by using transmission line method (TLM) with linear and circular contact pad geometries [26]. For linear TLM measurements, $75 \times 450 \mu \mathrm{m}^{2}$ contact pads were used with separations of 20, 40, 60, 80, 100, and 150 $\mu \mathrm{m}$. For 

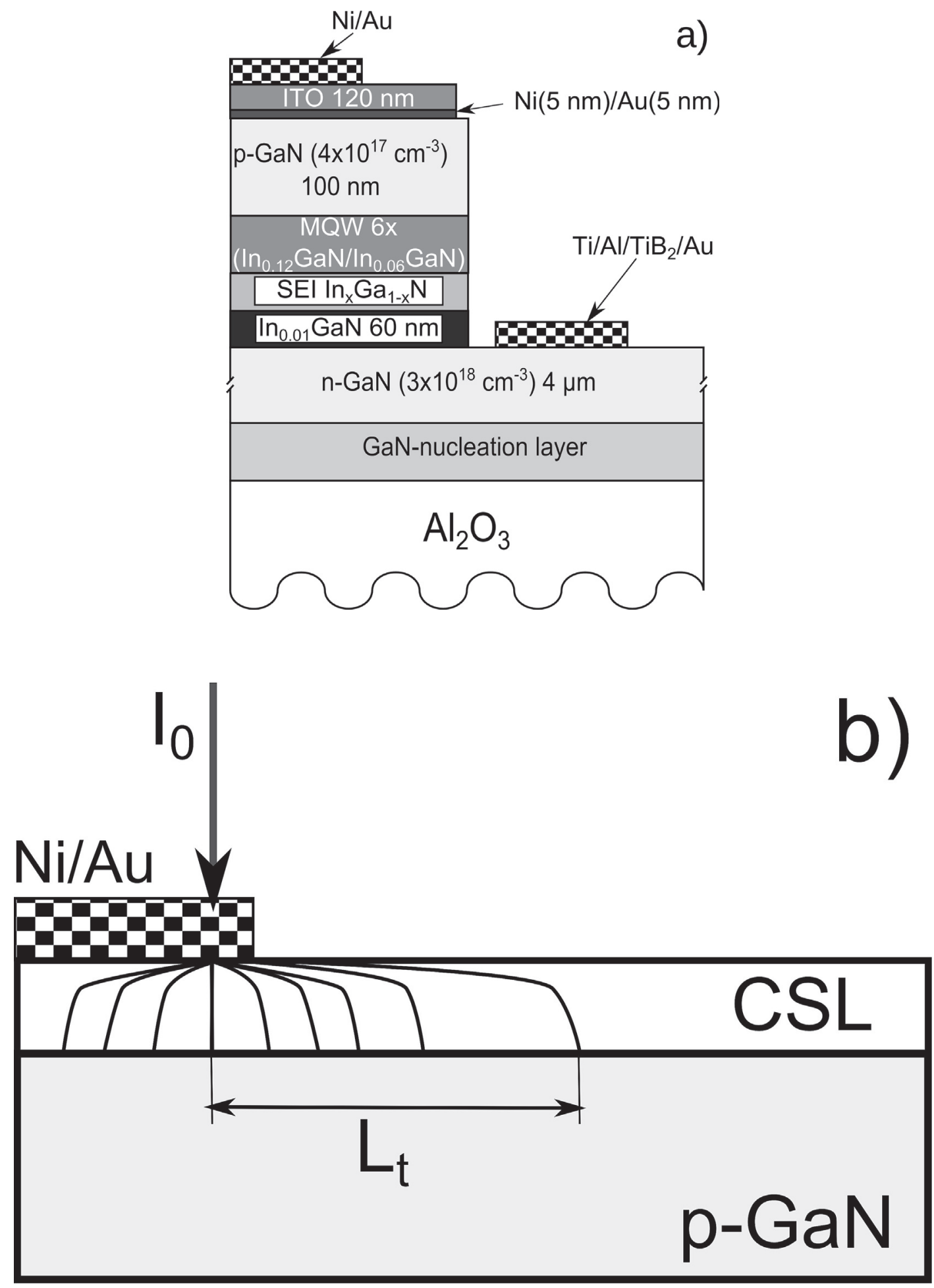

Fig. 1. Structure of fabricated InGaN/GaN LEDs (a) and schematic of current distribution in the CSL (b). 
circular TLM measurements inner contact pads had diameters of 50,80,100, and $150 \mu \mathrm{m}$ with constant ratio between outer and inner contact diameters equal to $2[26,27]$.

\section{Transfer length calculation}

The fabricated LED die structure and lateral current flow paths are shown in Fig. 1. For evaluation of current spreading efficiency in CSL, we use the transfer length that was proposed in papers $[26,28,29]$ which was then used for evaluating current spreading in LED structures in Ref. [30].

Current transfer length in CSL can be defined as the distance from the contact pad edge to the point in CSL where the current decreases down to $1 / \mathrm{e}$ of its maximum at the contact pad edge. This value can be calculated using the following equation [26]:

$$
L_{t}=\sqrt{\frac{\rho_{c} \cdot t}{\rho_{c s l}}},
$$

where $\rho_{c}$ - contact resistivity to the $\mathrm{p}$-GaN layer, $\rho_{c s l}$ - current spreading layer resistivity and $t$ - thickness of current spreading layer.

Current distribution in current spreading layer can be described using the proposed characteristic length via the equation:

$$
I_{x}=I_{0} \exp \left(-\frac{x}{L_{t}}\right)
$$

where, $I_{0}$ - current through metallic contact pad and $x$ - distance from the edge of contact pad.

To reiterate, we tested two different types of ohmic contacts to p-GaN cap layer of the LED structures. For the first type, ohmic contact was fabricated by sputtering $130 \mathrm{~nm}$ of ITO directly on p-GaN. Such an ohmic contact is typically characterized by its specific contact resistivity of $\sim 10^{-1} \Omega \mathrm{cm}^{2}$ after an RTA treatment [31]. After RTA in $\mathrm{O}_{2}$ at $700{ }^{\circ} \mathrm{C}$ for $1 \mathrm{~min}$, we achieved a contact resistivity of $\rho_{c}=8 \cdot 10^{-2} \Omega \cdot \mathrm{cm}^{2}$. Contacts of the second type were fabricated as follows: a Ni (5 nm)/Au (5 nm) stack was first deposited followed by RTA under $\mathrm{O}_{2}$ atmosphere at $500{ }^{\circ} \mathrm{C}$ for $5 \mathrm{~min}$, followed by ITO deposition. The resulting contact resistivity was $\rho_{c}=5 \cdot 10^{-3} \Omega \cdot \mathrm{cm}^{2}$. In both cases, the ITO layer resistivity was $\rho_{c s l}=5 \cdot 10^{-4} \Omega \cdot \mathrm{cm}$, consistent with reported values [5].

Results of transfer length calculations for resistivities and thicknesses used in this work for the current spreading layer are shown in Fig. 2. Fig. 2a represents the transfer length as a function of $\rho_{c} / \rho_{c s l}$. The measured values for $\rho_{c} / \rho_{c s l}$ for two different types of ohmic contacts, with and without the $\mathrm{Ni}(5 \mathrm{~nm}) / \mathrm{Au}(5 \mathrm{~nm})$ stack, provide transfer lengths of 114 and $456 \mu \mathrm{m}$, respectively. The dependence of the transfer length on the thickness of the current spreading layer is plotted in Fig. $2 \mathrm{~b}$ for both contact types. Current transfer length has a square root dependence on thickness of CSL, and thus increasing the CSL thickness does not increase $L_{t}$ noticeably but can indeed decrease the transparency of CSL and hence, the overall light output. Therefore, an increase in the current transfer length can be achieved through either increase in the specific contact resistivity or decrease in the resistivity of CSL. Increasing of the contact resistivity leads to an increase in the LED series resistance ( $\left.R_{S}\right)$ and thus degrades efficiency. The resistivity of CSL is limited by ITO material characteristics. Finally, if the size of the die is made smaller than $L_{t}$ to combat the poor current spreading, the series resistance increases.

\section{Results and discussion}

In this work, LED dies of square configuration (Type 1) and of interdigitated configuration (Type 2) were fabricated. Type 1a and 2a have just ITO (130 nm) as CSL and ohmic contact to $\mathrm{p}-\mathrm{GaN}$. Type $1 \mathrm{~b}$ and $2 \mathrm{~b}$ use Ni(5 nm)/Au (5 nm)/ITO(120 nm) as CSL and ohmic contact. Results of contact resistivity measurements and calculated transfer lengths for dies with different ohmic contacts to $\mathrm{p}-\mathrm{GaN}$ are presented in Table 1.

An optical microscope image of a typical LED die with square shape is presented in Fig. 3a. LED dies of Type 1a and 1b show the same emission wavelength with peak wavelength at $423 \mathrm{~nm}$ (Fig. 3b).

Fig. 4 shows representative I-V curves measured in the CW mode for LED dies of Type 1a and Type $1 \mathrm{~b}$. Series resistances for dies of Type 1a with sizes of $300 \times 300 \mu \mathrm{m}^{2}, 600 \times 600 \mu \mathrm{m}^{2}$, and $1000 \times 1000 \mu \mathrm{m}^{2}$ are $14.4 \Omega, 12.3 \Omega$ and $16.0 \Omega$, respectively (Table 2). One can note that, increasing the LED die area does not decrease the series resistance, as could be expected. Similar results are observed for the Type $1 \mathrm{~b}$ LEDs. Series resistances for small, middle, and large mesas of Type $1 \mathrm{~b}$ are $14.2 \Omega, 14.5 \Omega$ and $14.2 \Omega$, respectively (Table 2). These results suggest that series resistance is determined by current spreading rather than the mesa size. As a result, effective optical area of LED die is smaller than its physical area and is nearly the same for dies with different sizes because of poor current spreading. Dispersion in the active optical area between Type 1a and Type $1 \mathrm{~b}$ LEDs is determined by the difference in contact resistivity that in turn causes different transfer lengths in the current spreading layer. 


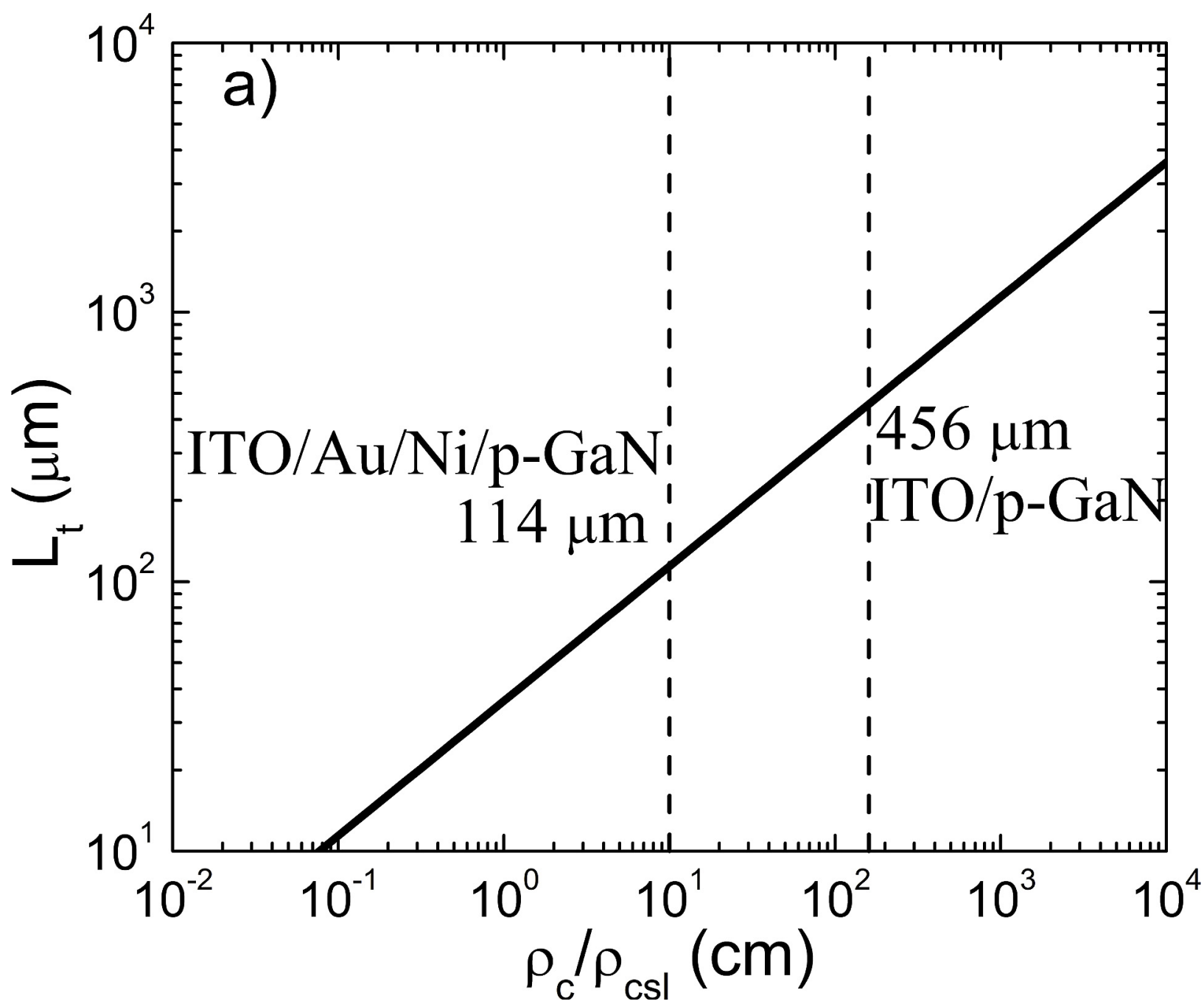

Fig. 2. Transfer length dependence on (a) the ratio between specific contact resistivity of p-type contact and resistivity of current spreading layer and (b) on the thickness of the CSL.

It is well known that defects and surface states at the side walls of mesas in GaN based epitaxial structures can lead to leakage currents that can be revealed in the I-V curves [1]. Non-zero current under reverse bias is an evidence of leakage. Under forward bias, leakage causes current flow below the expected turn-on voltage and is called the shunt current [1]. One can see in Fig. 4 that some of the diodes exhibit a small but non-negligible current flow at voltages smaller than the turn-on voltage, $V_{t}$. LEDs with leakage can be modeled by a parallel non-radiative current path that shunts the LED under test [1]. The corresponding equivalent circuit of the device is shown in the inset of Fig. 4, where $R_{s 1}$ is actual series resistance of the diode and $R_{p}$ is the parallel (shunt) resistance. Dynamic $R_{p}$ can be calculated through the slope of the $\mathrm{I}-\mathrm{V}$ curve in the region with $V<V_{t}$. Table 2 summarizing electrical properties associated with square-mesas LEDs indicates that in the Type 1a samples, the maximum $R_{p}$ value is lower than that in the Type $1 \mathrm{~b}$ devices. This is due to the larger effective area of Type 1a LEDs because of their larger CSL transfer length. As a result, larger numbers of defects are involved in the current flow process, leading to a larger shunting current. The coefficient $\alpha=I_{s} / I$ in Table 2 refers to the ratio of the current injected into of the active region of a given LED to the total current that flows through the LED die that also includes the current which bypasses the quantum wells and does not partake in light generation.

In the case of Type 1a LEDs with larger transfer lengths, as depicted in Fig. 5, the radiant intensity varies by a factor of 3 for the same current density among the devices with the same physical area. The devices with better current spreading in LED die lead to higher light emission intensity. If LED die dimensions were smaller than transfer length, current crowding and further increasing of LED series resistance would be observed. 


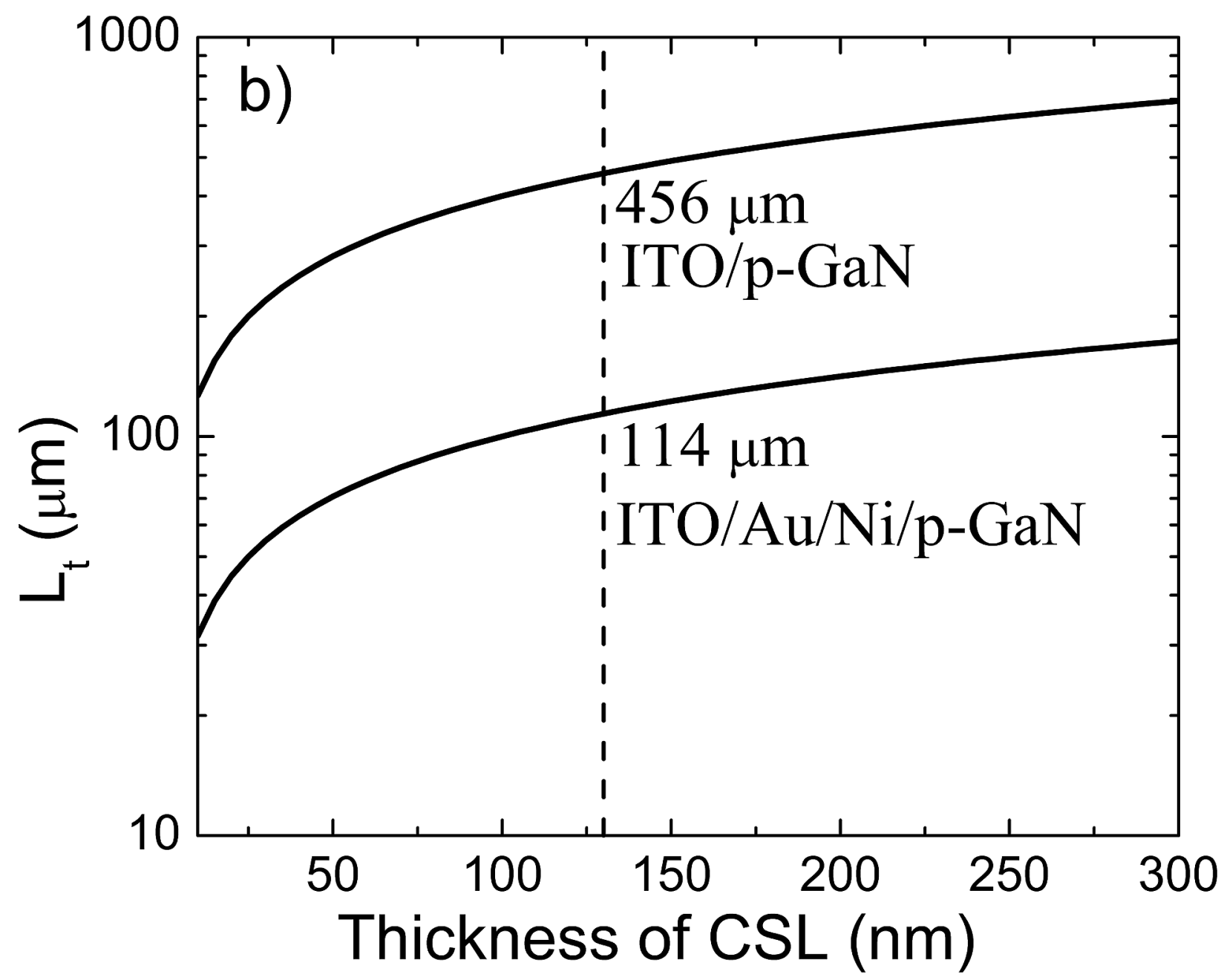

Fig. 2. (continued).

Table 1

Transfer lengths of LED dies with different ohmic contacts to p-GaN.

\begin{tabular}{llll}
\hline Die shapes & & Ohmic contact to p-GaN & $\rho_{c}\left(\Omega \cdot \mathrm{cm}^{2}\right)$ \\
\hline Square & Interdigitated & & $L_{t}(\mu \mathrm{m})$ \\
\hline Type 1a & Type 2a & ITO $(130 \mathrm{~nm})$ & $8 \cdot 10^{-2}$ \\
Type 1b & Type 2b & $\mathrm{Ni}(5 \mathrm{~nm}) / \mathrm{Au}(5 \mathrm{~nm}) / \mathrm{ITO}(120 \mathrm{~nm})$ & $5 \cdot 10^{-3}$ \\
\hline
\end{tabular}

One can evaluate the efficiency of LED chip using specific quantum efficiency that can be defined as the ratio of emitted photons to injected electrons divided by the LED die area [12]:

$$
\eta=\frac{P / h \nu}{I / q} \cdot \frac{1}{A}=\frac{P q}{I h \nu A}
$$

where $P$ is the emitted optical power, $I$ is the injected current, $A$ is the area of LED die, $q$ is the charge of electron, $h$ is the Planck constant and $\nu$ is the frequency of emitted light. The emitted power is, then:

$$
P=\eta h \nu \frac{I}{q} A
$$

One can see that the emitted optical power from LED is proportional to the injected current, the specific quantum efficiency of LED, and the area of LED die. To achieve the same optical power output would require the same optical area and current 


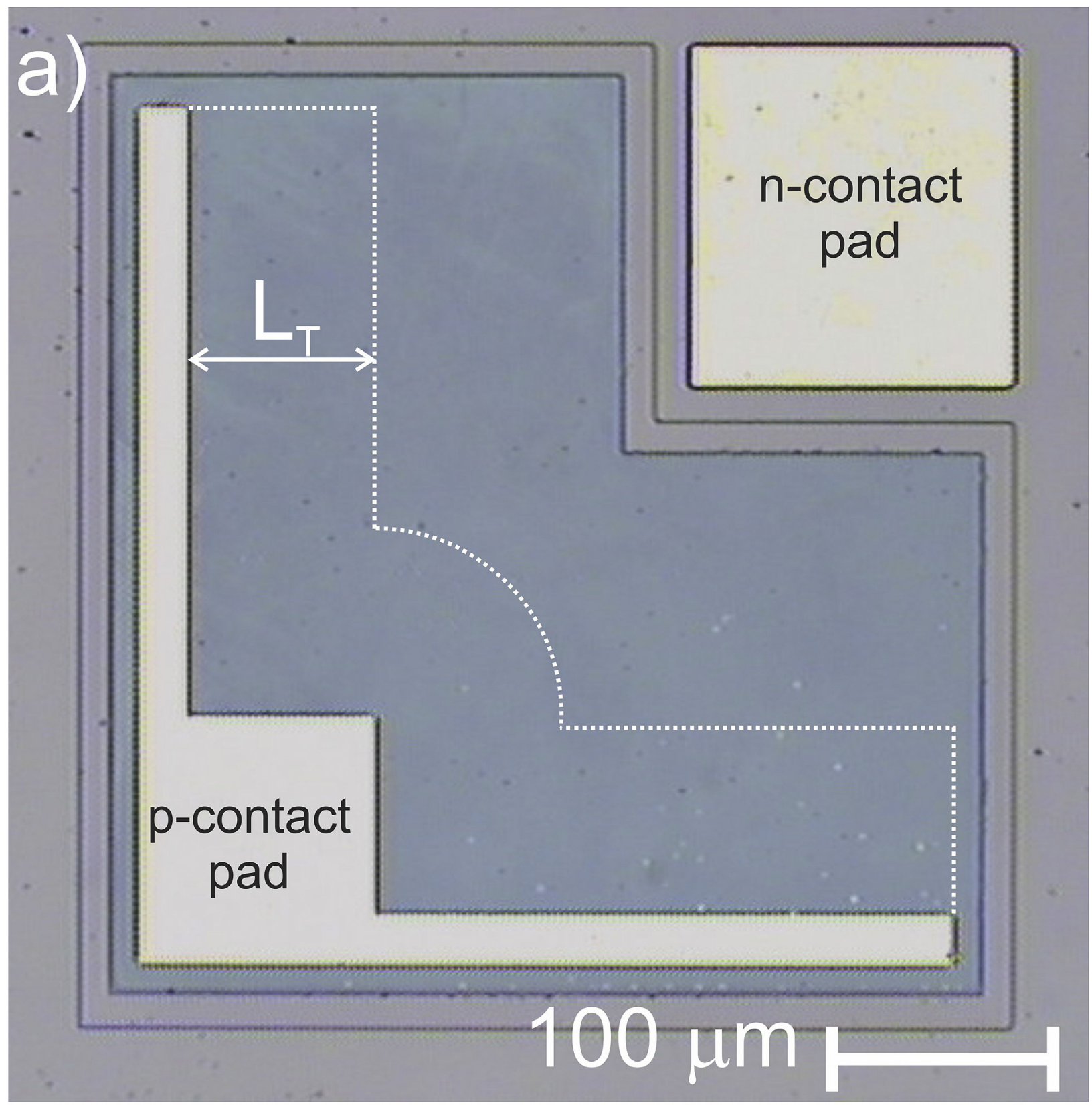

Fig. 3. (a) Light microscope image of LED die Type 1 and (b) its electroluminescence spectrum.

product, meaning increased area. Thus, it would be necessary to use different currents for LED dies with different areas fabricated on the same wafer to obtain the same power according to this equation. Namely, the ratio of currents should be equal to ratio of areas:

$$
\begin{aligned}
& \Delta_{1}=\frac{I_{2}}{I_{1}} ; \\
& \Delta_{2}=\frac{\left(A_{0}+A_{1}\right) \alpha_{1}}{\left(A_{0}+A_{2}\right) \alpha_{2}} ; \\
& \Delta_{1}=\Delta_{2} .
\end{aligned}
$$




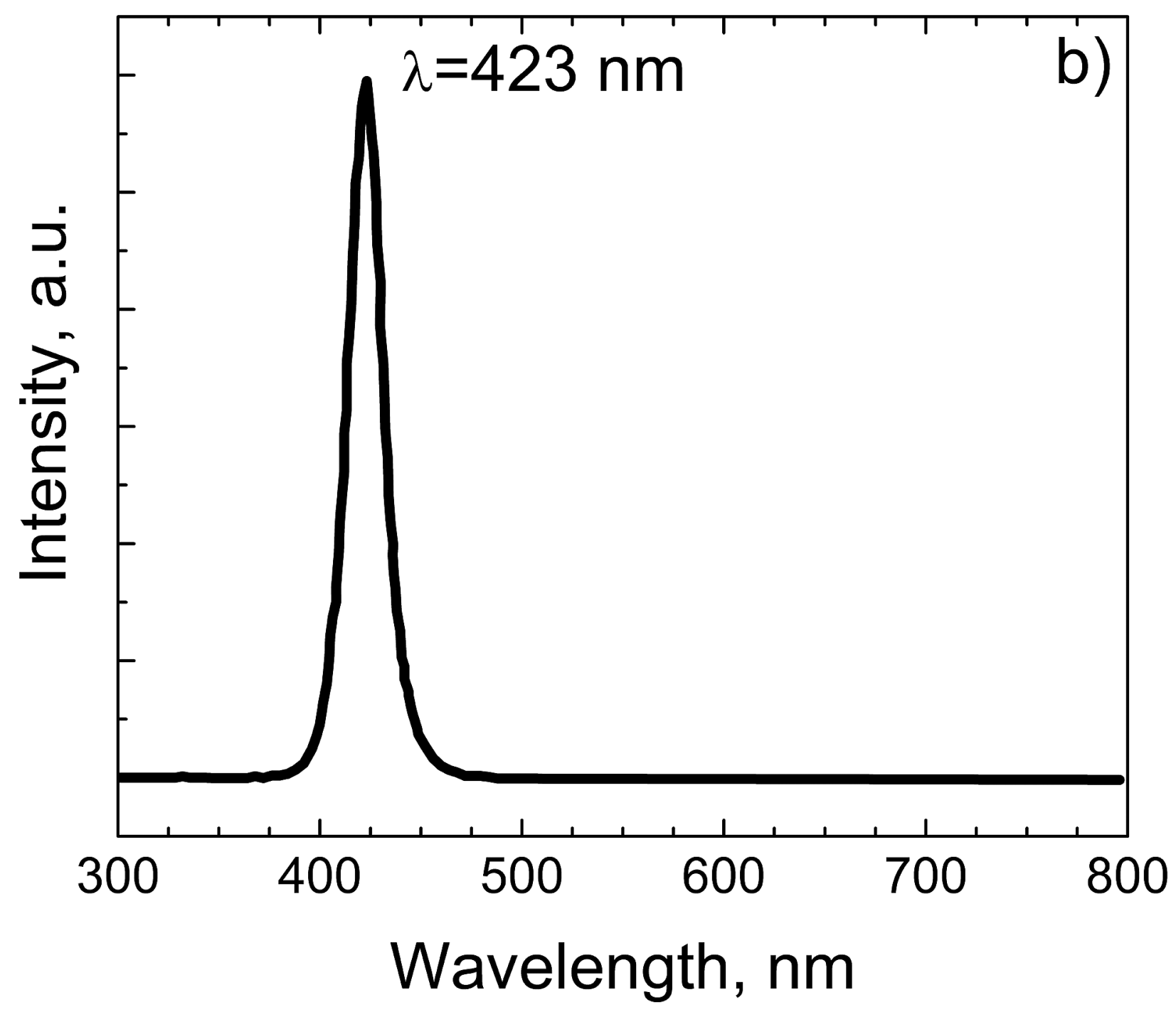

Fig. 3. (continued).

where $I_{1}$ and $I_{2}$ are currents used for getting of the same power of emitted light of LED chips of different area, $A_{0}$ is the area of contact pad, $A_{1}$ and $A_{2}$ are the areas of current spreading about the contact pads of respective die that is determined by transfer length in current spreading layer, $\alpha_{1}, \alpha_{2}$ - are coefficients that allow to take that into account part of current bypassing the radiative processes because of shunting current.

The optical power that is emitted by the LED die is proportional to measured radiant intensity of LEDs. Let us compare the current density that is injected into the LED structures and allows to emit light with the same radiant intensity from Type 1a and Type 1b LED dies (Fig. 5). For small-size LED dies, the ratio between the currents that is equal to ratio between the calculated current densities (Fig. 5) for the Type $1 \mathrm{a}$ and the Type $1 \mathrm{~b}$ die is equal to $\sim 2.6$. Assuming metal resistivity is equal to zero for the contact pads and taking into account the transfer length in the ITO layer, we can determine the ratio between the active optical area of LED chips with ITO and Ni/Au/ITO p-type contacts (Fig. 3a). The total area of the contact pad $A_{0}$ for small dies of Type 1 is equal to $1.4 \cdot 10^{-4} \mathrm{~cm}^{2}$. Areas that are determined by transfer length are $A_{1}=11.1 \cdot 10^{-4} \mathrm{~cm}^{2}$ and $A_{2}=4.28 \cdot 10^{-4} \mathrm{~cm}^{2}$ for LED dies of Type $1 \mathrm{a}$ and Type $1 \mathrm{~b}$, respectively. The ratio between the active areas of small LED dies is equal to 2.8 .

We proceeded with the same calculation for the other die sizes as well. The results are summarized in Table 3 for all three die sizes. In the case of medium size dies, the ratio between current densities is $\sim 2.4$. The calculated ratio between active area of Type $1 \mathrm{a}$ LED die and Type $1 \mathrm{~b}$ is equal to 2.3 in this case. For the large size dies, the ratio between current densities is equal to 


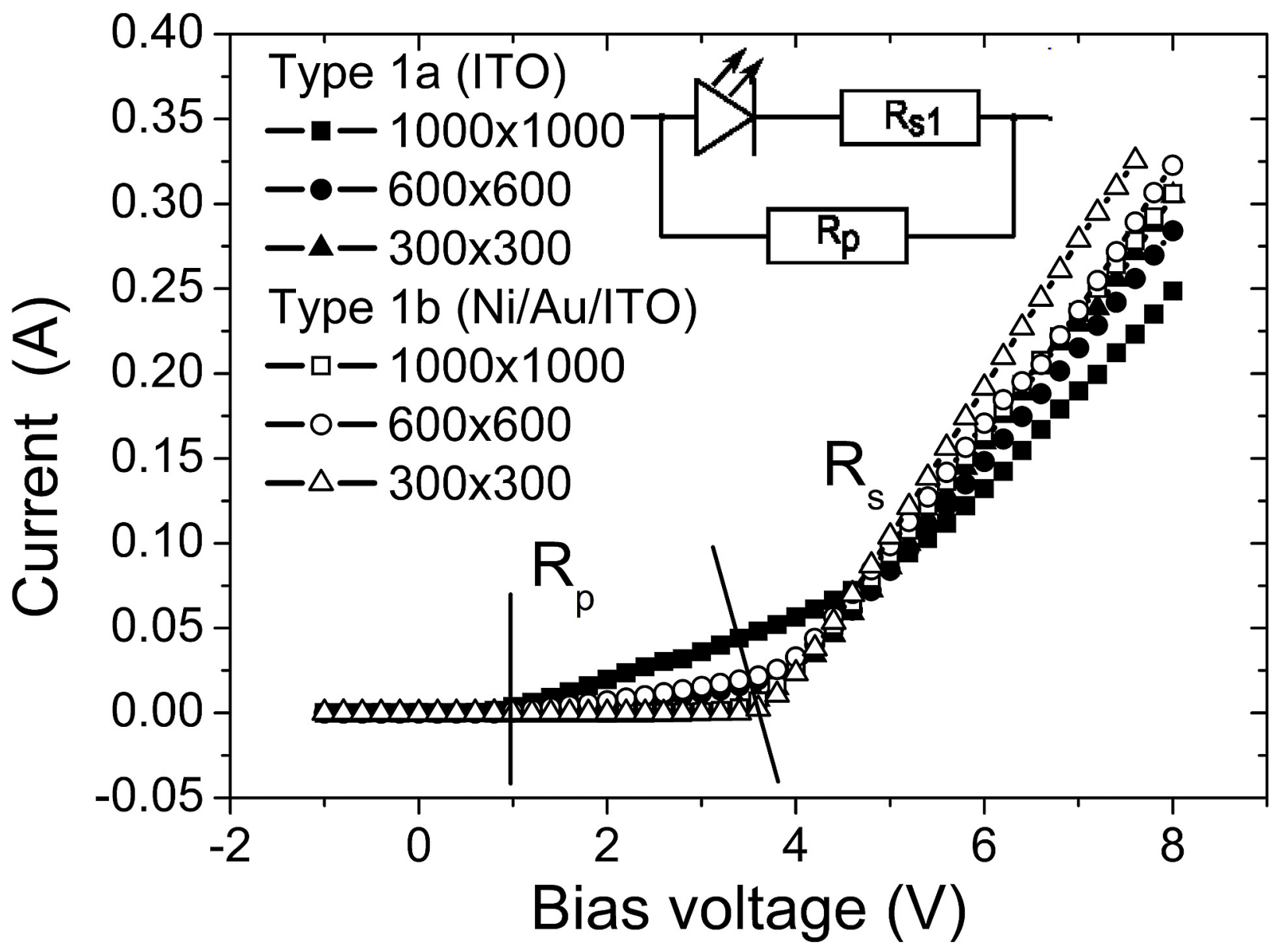

Fig. 4. I-V dependence of LEDs with ITO and Ni/Au/ITO ohmic contacts to p-GaN. The inset shows the device equivalent circuit.

1.5. The ratio between the active optical areas of these LEDs is equal to 2.6, however, with taking into account the shunt current, the ratio is found to be 1.8. The main contribution to the discrepancy between the ratios of active optical areas and current densities for the large LEDs is the shunting current that comprises 29\% of the total current in the LED with ITO ohmic contacts to p-GaN. One should keep in mind that, in reality, the resistance of metal contact pads is not zero, and the calculation presented above allows only the determination of the ratio between active areas but does not make it possible to draw a conclusion about the actual values of active areas.

Our results show that, for InGaN/GaN LED dies of the conventional square shape and given size, the same series resistance can be observed even with ohmic contacts with different resistivities to p-GaN. However, to achieve the same optical power emitted from LED dies of Type $1 \mathrm{a}$ and Type $1 \mathrm{~b}$, the injection current has to be different. The ratio of these currents is equal to the ratio between the active optical areas of LED dies. These active optical areas can be determined by the transfer length in CSL that depends on the ratio between the ohmic contact resistivity and resistivity of CSL.

To overcome current spreading limitations and use the area of LEDs more effectively, we use an LED die with interdigitated electrode configuration of similar size with the previous LED to improve light emission. In this vein, LED dies with ITO $(130 \mathrm{~nm}) / \mathrm{p}-\mathrm{GaN}$ (Type 2a) and ITO(120 nm)/Au(5 nm)/Ni(5 nm)/p-GaN (Type $2 \mathrm{~b}$ ) ohmic contacts were fabricated. The distance from the contact pad edge to the edge of p-n junction is $50 \mu \mathrm{m}$ (Type 2) (Fig. 6a). Consequently, the active area is the same as the real area because the transfer length for current spreading layers with ITO and Ni/Au/ITO as ohmic contacts to pGaN layer is larger than this characteristic distance (Table 1 ). LEDs of Type $2 a$ and Type $2 b$ were characterized by the same spectra. Representative LED spectrum of these LEDs is shown in Fig. 6b.

Analysis of $\mathrm{I}-\mathrm{V}$ characteristics shows that series resistance decreases with increasing LED die area, thus indicating that effective areas of dies depend on the physical area of the dies (Fig. 7). Series resistances of Type 2a samples are equal to $35 \Omega$, $17.9 \Omega$, and $15.8 \Omega$ for $360 \times 360 \mu \mathrm{m}^{2}, 660 \times 660 \mu \mathrm{m}^{2}$, and $1060 \times 1060 \mu \mathrm{m}^{2}$ dies, respectively. Series resistances of Type $2 \mathrm{~b}$ samples are $36.4 \Omega, 20.1 \Omega$ and $19.3 \Omega$ for the corresponding die sizes (Table 4 ). 
Table 2

Electrical properties of LEDs of Type 1.

\begin{tabular}{|c|c|c|c|c|c|c|c|c|}
\hline \multirow[t]{2}{*}{ Die size $\left(\mu \mathrm{m}^{2}\right)$} & \multicolumn{4}{|c|}{ Type 1a (ITO/p-GaN) } & \multicolumn{4}{|c|}{ Type 1b (ITO/Au/Ni/p-GaN) } \\
\hline & $I_{\text {leak }}(\mathrm{mA})$ & $R_{S}(\Omega)$ & $R_{p}(\Omega)$ & $\alpha(\%)$ & $I_{\text {leak }}(\mathrm{mA})$ & $R_{S}(\Omega)$ & $R_{p}(\Omega)$ & $\alpha(\%)$ \\
\hline $300 \times 300$ & $4.4 \cdot 10^{-2}$ & 14.4 & 1963 & 99.3 & $1.9 \cdot 10^{-2}$ & 14.2 & 51210 & 99.9 \\
\hline $600 \times 600$ & $1.8 \cdot 10^{-2}$ & 12.3 & 147 & 91.6 & $2.7 \cdot 10^{-2}$ & 14.5 & 126 & 88.5 \\
\hline $1000 \times 1000$ & $1.8 \cdot 10^{-3}$ & 16.0 & 56 & 71.2 & $8.6 \cdot 10^{-2}$ & 14.2 & 2297 & 99.4 \\
\hline
\end{tabular}

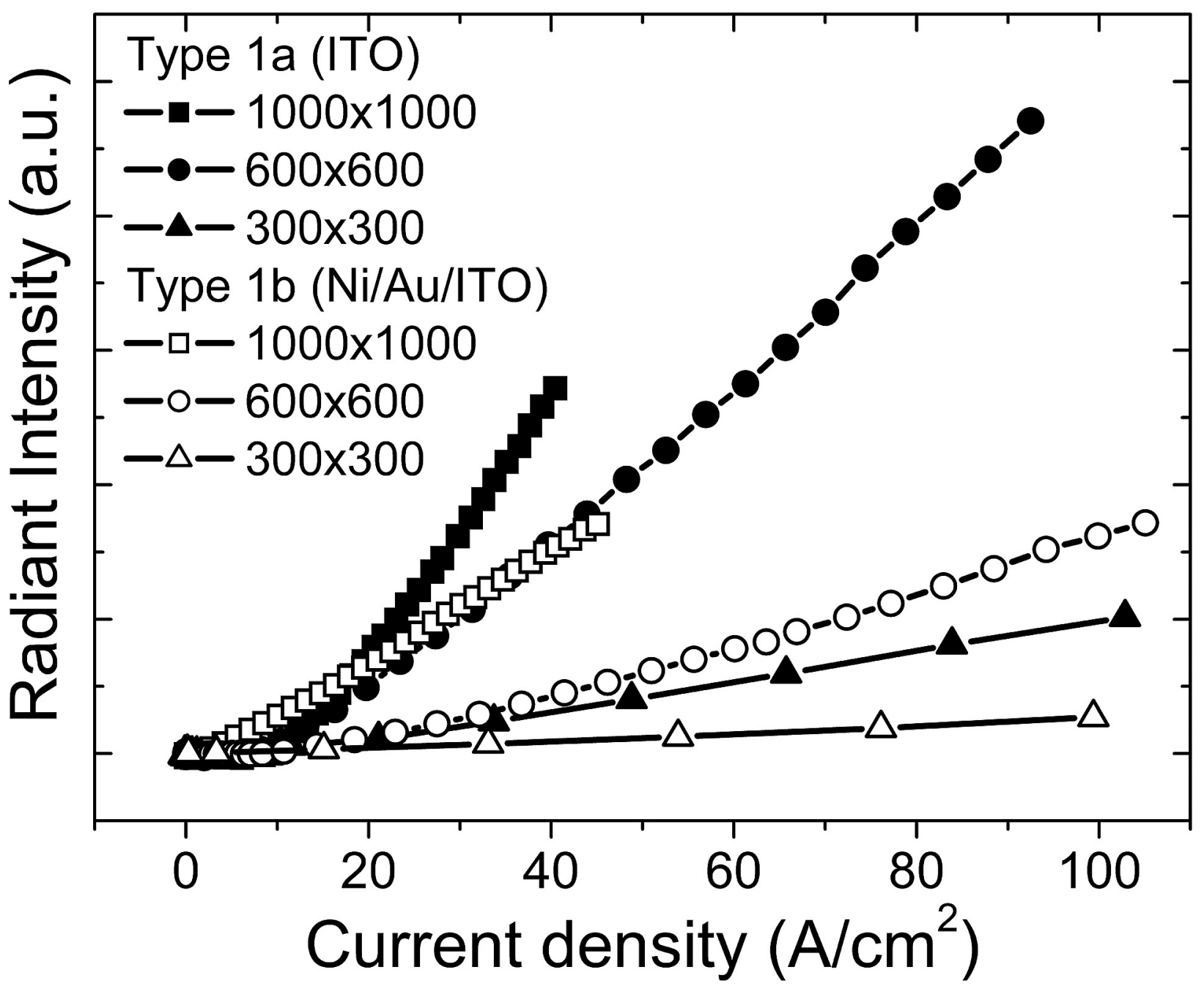

Fig. 5. Radiant intensity dependence on current density of LEDs of Type $1 \mathrm{a}$ and Type $1 \mathrm{~b}$ in the CW mode.

In the LED die of Type 2 configuration with interdigitated electrode patterns, the series resistance is higher than that of Type 1 LEDs, especially for the small die structures. This can be caused by smaller areas of contact to $n-G a N$ and contact pad to CSL in Type 2 LEDs than in Type 1 LEDs. This difference exceeds a factor of two for contacts to n-GaN for small LED dies $\left(1.4 \cdot 10^{-4} \mathrm{~cm}^{2}\right.$ for Type 1 and $0.64 \cdot 10^{-4}$ for Type 2). The areas of contact pads to CSL in small dies are $1.4 \cdot 10^{-4} \mathrm{~cm}^{2}$ and

Table 3

Ratios of active areas and currents at constant radiant intensities of LED dies of Type1a and Type $1 \mathrm{~b}$.

\begin{tabular}{llllll}
\hline Die size $\left(\mu \mathrm{m}^{2}\right)$ & $A_{0} \cdot 10^{-4}\left(\mathrm{~cm}^{2}\right)$ & $A_{1} \cdot 10^{-4}\left(\mathrm{~cm}^{2}\right)$ & $A_{2} \cdot 10^{-4}\left(\mathrm{~cm}^{2}\right)$ & $\Delta_{1}$ & 2.8 \\
\hline $300 \times 300$ & 1.4 & 11.1 & 4.28 & 2.6 & 2.4 \\
$600 \times 600$ & 4.5 & 25.9 & 9.6 & 2.3 \\
$1000 \times 1000$ & 6.9 & 58.9 & 18.7 & 1.5 \\
\hline
\end{tabular}




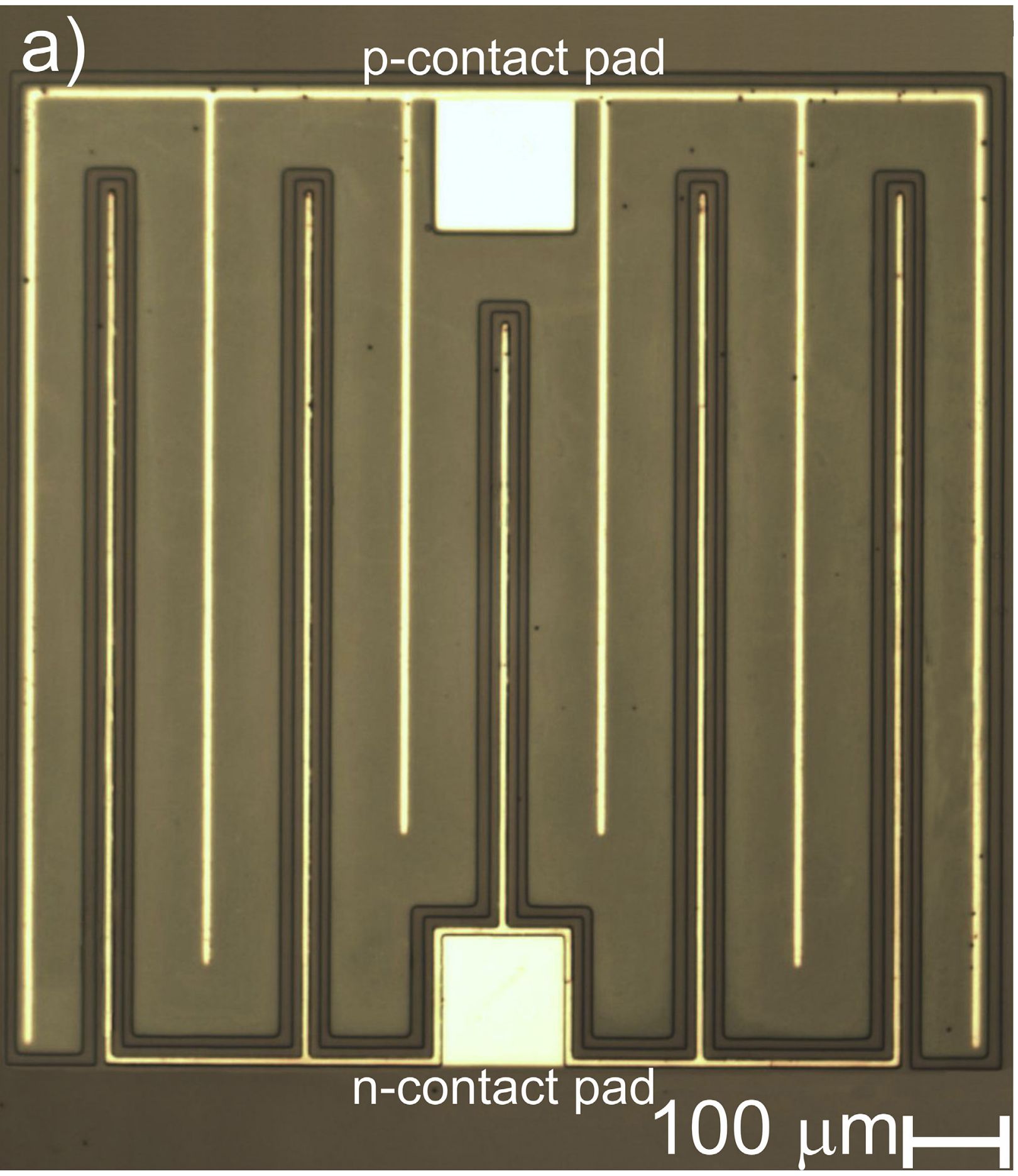

Fig. 6. Light microscope image of Type 2 LED die with interdigitated contact pattern (a), and the LED electroluminescence spectrum (b).

$0.94 \cdot 10^{-4} \mathrm{~cm}^{2}$ for Type 1 and Type 2 LEDs, respectively. For the medium LED dies, the areas are $4.4 \cdot 10^{-4} \mathrm{~cm}^{2}$ and $3.8 \cdot 10^{-4} \mathrm{~cm}^{2}$ for contacts to $\mathrm{n}-\mathrm{GaN}$ and $4.5 \cdot 10^{-4} \mathrm{~cm}^{2}$ and $2.6 \cdot 10^{-4} \mathrm{~cm}^{2}$ for contact pads to CSL for Type 1 and Type 2 LEDs, respectively. The die area that can be used for light extraction from small dies increased from $62 \%$ to $81 \%$ because of a change in contact area. 


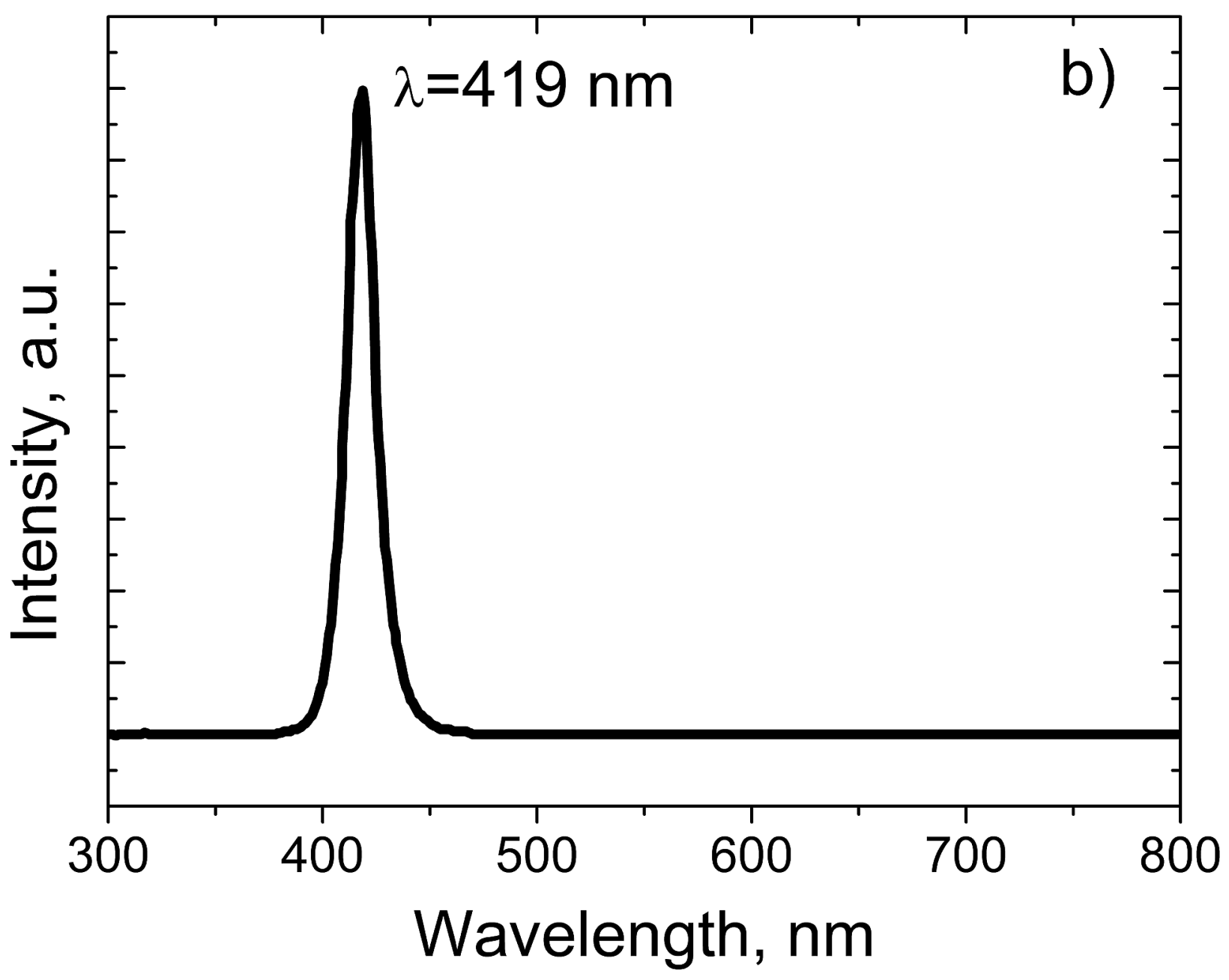

Fig. 6. (continued).

Higher series resistance in samples with lower resistivity of contacts to p-GaN is also unexpected. Resistance of contact to p-GaN is smaller for Type 2b LED dies than that for Type 2a LED dies (Table 1); therefore, the series resistances should have the same relationship (Table 4). Type 2a LEDs with ITO contacts are characterized by larger leakage currents that affect the I-V characteristics. This can be understood by taking into account the transfer length for these samples. As a result of larger contact resistivity, the transfer length of Type 2a LED is 8 times larger than the characteristic distance between the contact pad and the edge of p-n junction. Therefore, the probability of surface leakage through mesa walls as well as sampling of larger number of defects is much higher for Type 2a LEDs than those in the case of Type $2 b$ LEDs. The same mechanism for current flow may be responsible for higher parallel current flow that manifests itself at sub-threshold voltages in forward bias I-V characteristics. Results of parallel resistance $\left(R_{p}\right)$ calculations are summarized in Table 4 . One can see that parallel resistance is much smaller for LED Type 2a indicating much higher probability of leakage current flow.

The series resistance calculated from the slope of the measured $\mathrm{I}-\mathrm{V}$ characteristics for the above-threshold region includes the parallel resistance as well. To derive the actual series resistance of LED die, the parallel resistance should be subtracted using the following equation:

$$
R_{s 1}=\frac{R_{p} R_{S}}{R_{p}-R_{S}}
$$

After subtraction of the parallel resistance, the real series resistance $\left(R_{s 1}\right)$ of Type $2 \mathrm{~b}$ LED dies is much lower compared with Type 2a dies (Table 4) which correlates with the values of contact resistivities to p-GaN. In the case of small dies, the ratio 


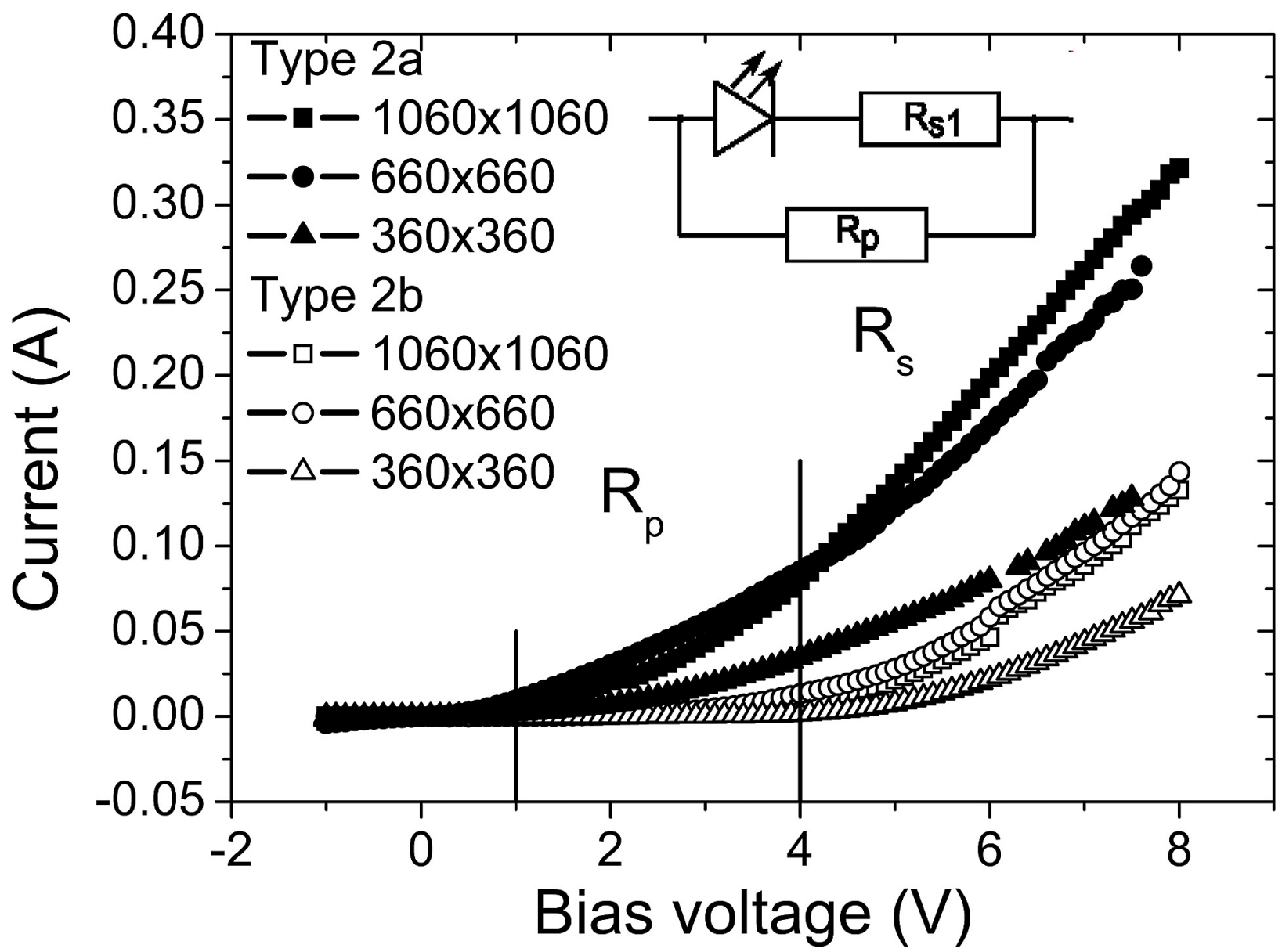

Fig. 7. Radiant intensity of LEDs of Type 2 with ITO and Ni/Au/ITO ohmic contacts to the p-GaN.

Table 4

Electrical parameters of LEDs of Type 2.

\begin{tabular}{|c|c|c|c|c|c|c|c|c|c|c|}
\hline \multirow{2}{*}{ Die size $\left(\mu \mathrm{m}^{2}\right)$} & \multicolumn{5}{|c|}{ Type 2a (ITO/p-GaN) } & \multicolumn{5}{|c|}{ Type 2b (ITO/Au/Ni/p-GaN) } \\
\hline & $I_{\text {leak }}(\mathrm{mA})$ & $R_{S}(\Omega)$ & $R_{p}(\Omega)$ & $R_{s 1}(\Omega)$ & $\alpha(\%)$ & $I_{\text {leak }}(\mathrm{mA})$ & $R_{S}(\Omega)$ & $R_{p}(\Omega)$ & $R_{s 1}(\Omega)$ & $\alpha(\%)$ \\
\hline $360 \times 360$ & 1.3 & 35 & 73.4 & 66.9 & 52.3 & $1.7 \cdot 10^{-3}$ & 36.4 & 2187 & 37 & 98.3 \\
\hline $660 \times 660$ & 4 & 17.9 & 37.6 & 34.2 & 52.4 & $2.7 \cdot 10^{-2}$ & 20.1 & 200 & 22.3 & 90.0 \\
\hline $1060 \times 1060$ & 2.9 & 15.8 & 32.9 & 30.4 & 52.0 & 0.1 & 19.3 & 319 & 20.5 & 94.0 \\
\hline
\end{tabular}

between the series resistances of the Type $2 \mathrm{a}$ and the Type $2 \mathrm{~b}$ die is close to 2 . Measurements of the radiant intensity also show that light intensity for Type 2b LEDs is 10 times higher (Fig. 8) for the same current density.

For LED dies of small size, 2.1 times higher current is required for Type 2a LED chip to produce the same radiant intensity as that for the Type $2 \mathrm{~b}$ chip. These ratios are 1.8 and 1.6 for the medium- and the large-size chips, respectively. The role of the shunting current and area can be evaluated through equation (5). In this case, the effective areas will be the same so the ratio of currents should be equal to the ratio of $\alpha$ coefficients.

The ratios are 1.9, 1.7 and 1.8 for the LED chips of small, medium, and large size, respectively (Table 5). The ratios are comparable with the corresponding current ratios, which indicate that the shunt currents are mainly responsible for the observed difference in the values of current required to produce the same emission power from the Type 2a and Type 2b LEDs.

Measured data for Type 2 LED chips show that, in the case of transfer length comparable to the distance between the contact pad and the edge of p-n junction, passivation of mesa walls is important for eliminating the leakage currents. One can see from Fig. 9 that Type 2b LEDs have higher radiant intensity compared to conventional square LED dies of Type 1a. For the same current densities, the improvement is $43 \%$ for small LED dies, and it reaches up to $140 \%$ for the large dies. However, in 


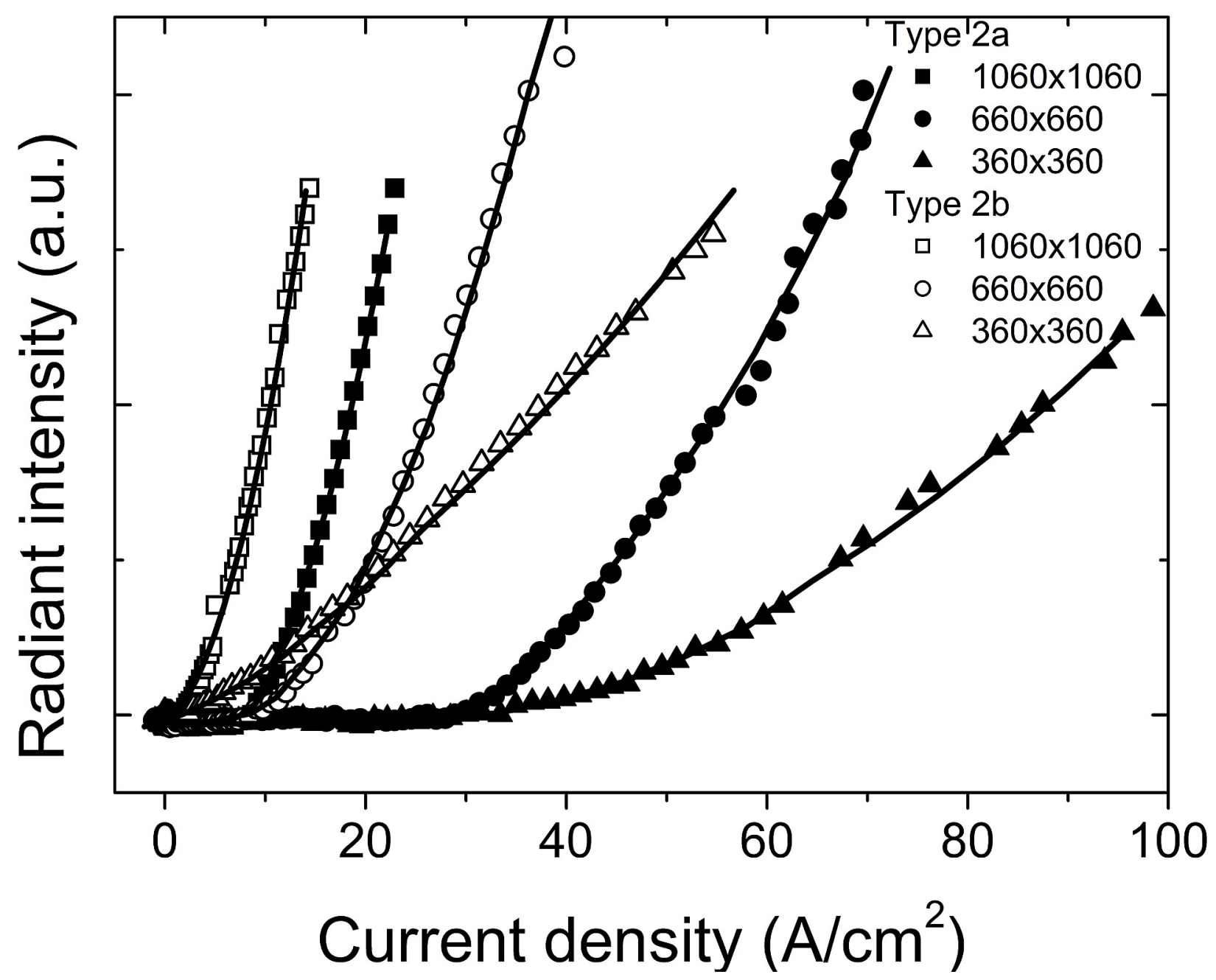

Fig. 8. Radiant intensity dependence on current density of Type 2 LEDs with interdigitated configuration of contacts.

Table 5

Ratios of active areas and currents constant radiant intensities of LED dies of Type 2a and Type $2 \mathrm{~b}$.

\begin{tabular}{lll}
\hline Die size $\left(\mu \mathrm{m}^{2}\right)$ & $I_{1} / I_{2}$ & $\alpha_{2} / \alpha_{1}$ \\
\hline $360 \times 360$ & 2.1 & 1.9 \\
$660 \times 660$ & 1.8 & 1.7 \\
$1060 \times 1060$ & 1.6 & 1.8 \\
\hline
\end{tabular}

the case of medium size die, the Type 1a LEDs produce $94 \%$ higher radiant intensity than the Type $2 \mathrm{~b}$ ones. This can be explained by higher leakage in LED dies of Type 2. Using passivation, one can achieve a greater improvement of radiant intensity for the Type 2 medium size LED dies. Moreover, the passivation should improve further the efficiency for small and large Type 2 LEDs. Moreover, we need take into account the fact that the active area is smaller than the total area of Type 1 LED dies. Therefore, their real current density is actually much higher for the same radiant intensity as in the case of Type 2 devices. This makes the performance of Type 2 LEDs even better than it appears from an initial inspection of the radiant intensity vs. the current density dependences.

To verify the validity of the above conclusion, we fabricated large-size Type $2 \mathrm{~b}$ LED chips with side-wall passivation with $250 \mathrm{~nm}$ thick $\mathrm{SiO}_{2}$. These LED dies exhibited a low series resistance of $12.8 \Omega$ and low shunt current because their 


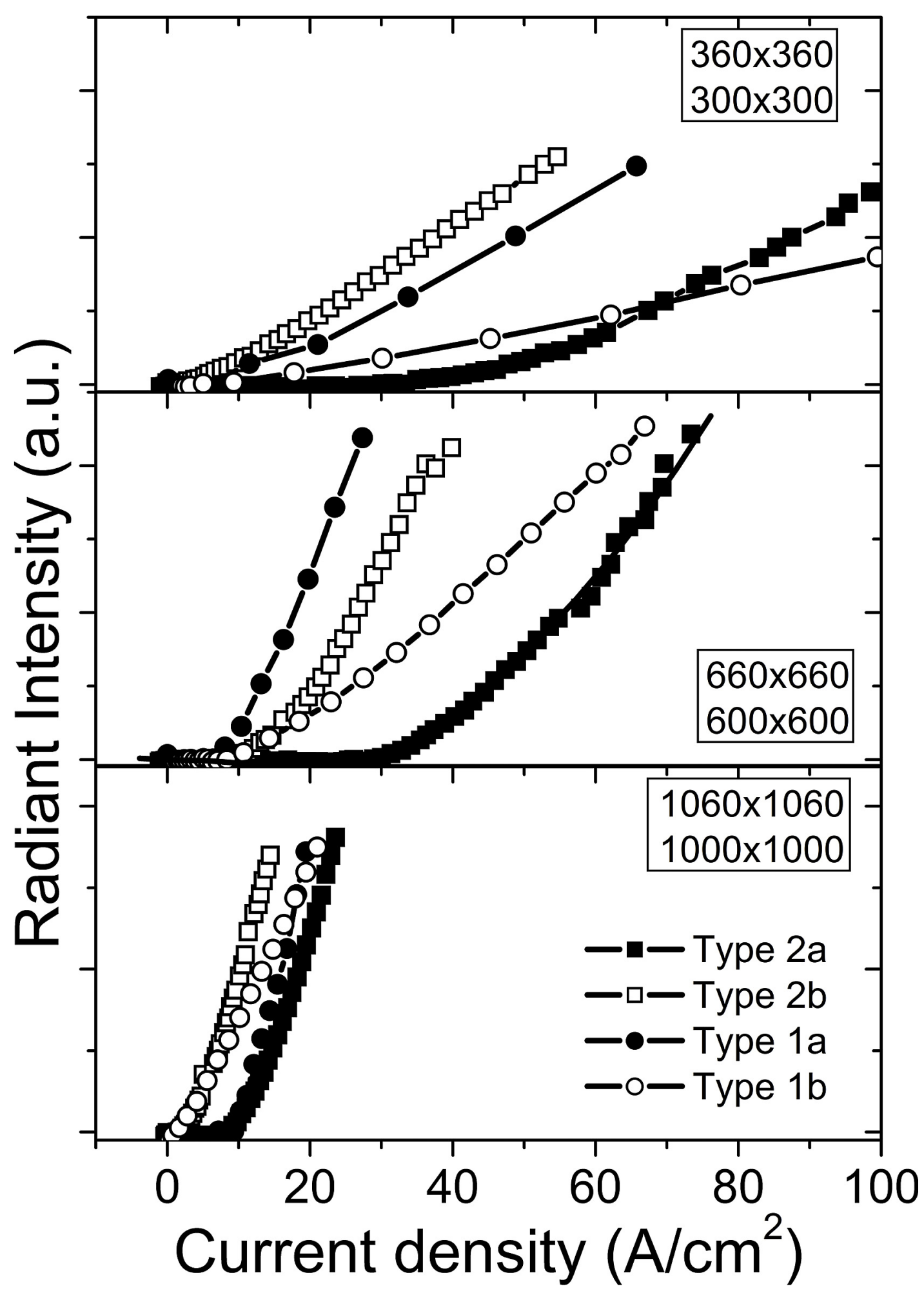

Fig. 9. Radiant intensity vs. current density for the Type 1 and Type 2 LED dies. 


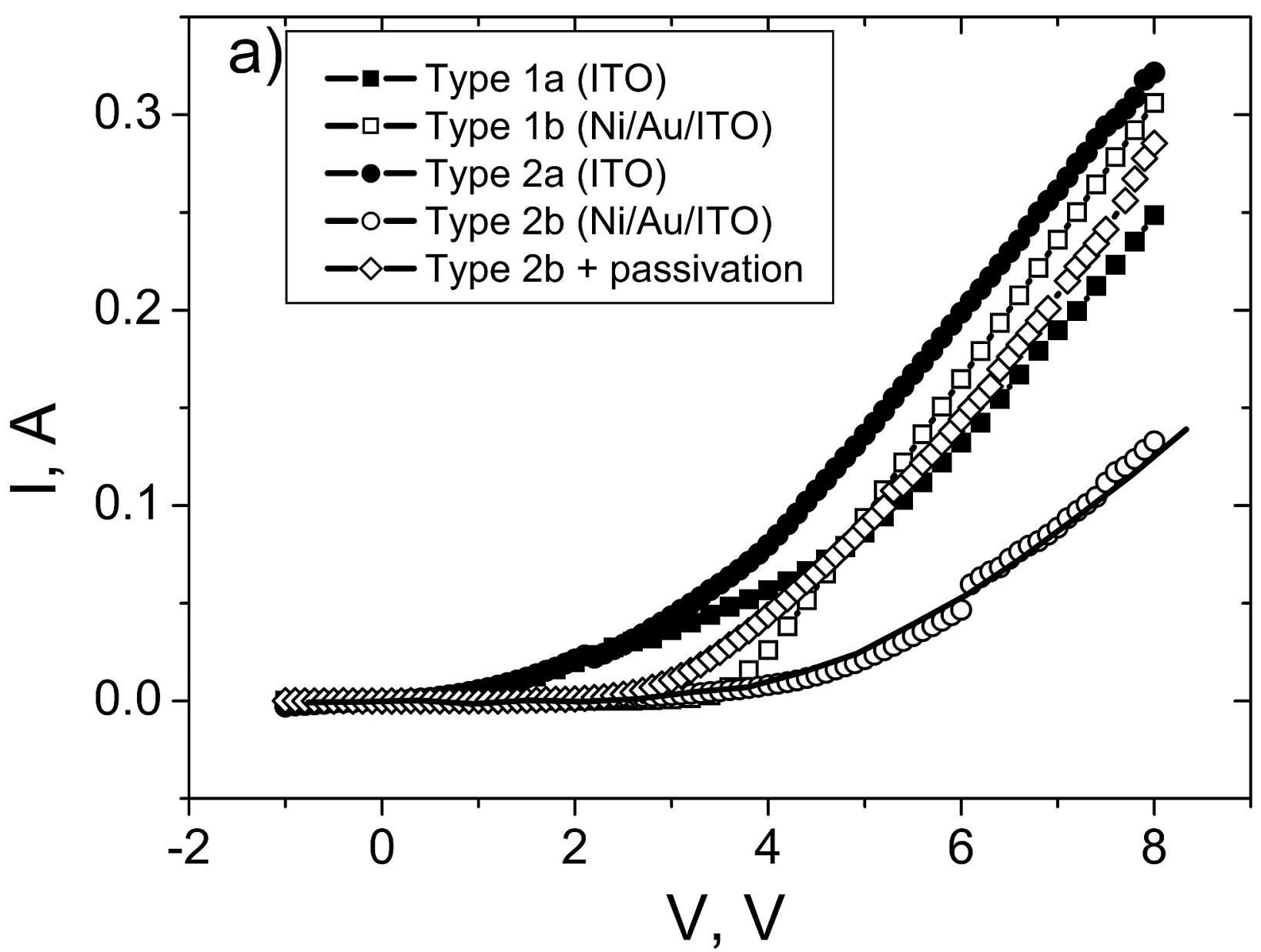

Fig. 10. I-V characteristics (a) and radiant intensity (b) vs. current density for large-size LED chips of Type 1a, 1b, 2a, $2 \mathrm{~b}$, and passivated Type $2 \mathrm{~b}$.

parallel resistance of $800 \Omega$ is much higher than the series resistance. As a result these devices showed a low leakage current of $6.1 \cdot 10^{-2} \mathrm{~mA}$ at $-1 \mathrm{~V}$ [Fig. $10(\mathrm{a})$ ]. This value is 1.6 times smaller than of LED $2 \mathrm{~b}(0.1 \mathrm{~mA})$ and 48 times smaller than for LED 2a (2.9 mA) (Table 4). As evident from Fig. 10 (b), the radiant intensity dependence on the current density is similar to that of the Type 2b LED but higher current densities and higher corresponding radiant intensities can be achieved for the passivated dies. As a result, the radiant intensity is nearly 1.5 times higher for the passivated Type $2 \mathrm{~b}$ LEDs compared to the Type 1a devices.

\section{Conclusions}

We have shown that the active area of InGaN/GaN LED with top p-GaN contact layer depends on the efficiency of current spreading in the current spreading layer. Increasing the die dimensions beyond the current spreading distance does not decrease the series resistance and does not improve LED brightness. We propose a current transfer length as a characteristic parameter quantifying current spreading. We have shown that the transfer length in the current spreading layer affects the efficiency of LED dies.

Unlike the previous models, we took into account the finite resistivity of the Indium Tin Oxide (ITO) current spreading layer (CSL) and show that the optical power of light emitted from an LED chip is proportional to its effective optical area and current that is injected to the active regions. Employment of LED dies with interdigitated electrodes, where the distance between the contacts pad on CSL and the edge of p-n junction is equal to the CSL transfer length, allows an increase of the LED chip effective area and makes it equal to the real area of die. As a result, the optical power emitted from an LED can be increased without current increasing, thus improving the LED efficiency. In addition, we demonstrated that, in order to 


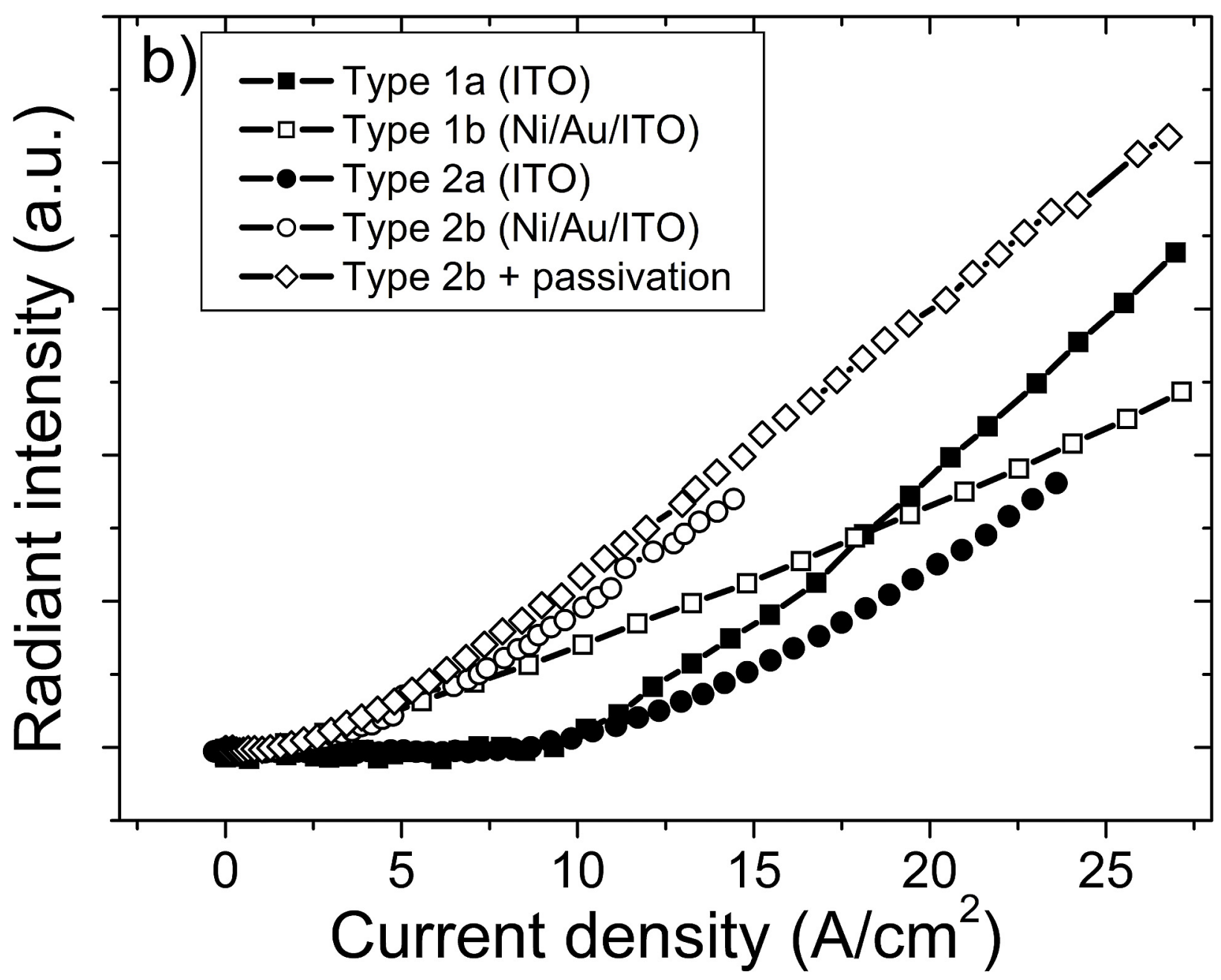

Fig. 10. (continued).

eliminate shunting currents, the passivation of LED edges is required when the distance between contact pads and the edge of LED die is equal or shorter than the CSL spreading length.

\section{Acknowledgements}

This work has been performed with the support of TÜBITAK (Grant no: 113G042). Ismail Altuntaş, acknowledges the Ph.D. Grant support from the Scientific and Technological Research Council of Turkey (TUBITAK).

We thank to UNAM-National Nanotechnology Research Center at Bilkent University for the use of some equipment.

\section{References}

[1] E.F. Schubert, Light-emitting Diodes, 2-nd ed, Cambridge University Press, Cambridge, 2006.

[2] D. Annaig, G. Graziella, D. Gerard, Gallium nitride bulk crystal growth processes: a review, Mat. Sci. Eng. R. 50 (2006) $167-194$.

[3] M. Levinstein, S. Rumyantsev, M. Shur, Handbook Series on Semiconductor Parameters, vol. 1, 2, World Scientific, London, 1996, p. 1999.

[4] W. Lu, D. Aplin, A.R. Clawson, P.K.L. Yu, Effects of the gas ambient in thermal activation of Mg-doped p-GaN on Hall effect and photoluminescence, J. Vac. Sci. Technol. A 31 (2013) 011502.

[5] D.S. Ginley, Handbook on Transparent Conductors, Springer Science\& Business Media, New York, 2010.

[6] T. Minami, Transparent conducting oxide semiconductors for transparent electrodes, Semicond. Sci. Technol. 20 (2005) S35-S44.

[7] X. Guo, E.F. Schubert, Current crowding and optical saturation effects in GaInN/GaN light-emitting diodes grown on insulating substrates, Appl. Phys. Lett. 78 (2001) 3337-3339.

[8] X. Guo, E.F. Schubert, Current crowding in GaN/InGaN light emitting diodes on insulating substrates, J. Appl. Phys. 90 (2001) $4191-4195$.

[9] G.H.B. Thompson, Physics of Semiconductor Laser Devices, Wiley, New York, 1980.

[10] H. Kim, J.-M. Lee, C. Huh, S.-W. Kim, D.-J. Kim, S.-J. Park, H. Hwang, Modeling of a GaN-based light-emitting diode for uniform current spreading, Appl. Phys. Lett. 77 (2000) 1903-1904. 
[11] H. Kim, S.-J. Park, H. Hwang, Effects of current spreading on the performance of GaN-based light-emitting diodes, IEEE Trans. El. Dev. 48 (2001) $1065-1069$.

[12] H. Kim, S.-J. Park, H. Hwang, Design and fabrication of highly efficient GaN-based light-emitting diodes, IEEE Trans. El. Dev. 49 (2002) 1715-1722.

[13] H. Kim, J. Cho, J. Wook Lee, S. Yoon, H. Kim, C. Sone, Y. Park, T.-Y. Seong, Consideration of the actual current-spreading length of GaN-based lightemitting diodes for high-efficiency design, IEEE J. Quant. El 43 (2007) 625-632.

[14] H. Kim, J. Cho, J. Wook Lee, S. Yoon, H. Kim, C. Sone, Y. Park, T.-Y. Seong, Measurements of current spreading length and design of GaN-based light emitting diodes, Appl. Phys. Lett. 90 (2007) 063510.

[15] J. Lv, C. Zheng, S. Zhou, F. Fang, S. Yuan, Highly efficient and reliable high power InGaN/GaN LEDs with 3D patterned step-like ITO and wavy sidewalls, Phys. Status Solidi A 213 (2016) 1181-1186.

[16] S. Zhou, S. Yuan, Y. Liu, L. Jay Guo, S. Liu, H. Ding, Highly efficient and reliable high power LEDs with patterned sapphire substrate and strip-shaped distributed current blocking Layer, Appl. Surf. Sci. 355 (2015) 1013-1019.

[17] M. Oh, W.-Y. Jin, H.J. Jeong, M.S. Jeong, J.-W. Kang, H. Kim, Silver Nanowire transparent conductive electrodes for high-efficiency III-nitride lightemitting diodes, Sci. Rep. 5 (2015) 13483.

[18] S. Huang, B. Fan, Z. Chen, Z. Zheng, H. Luo, Z. Wu, G. Wang, H. Jiang, Lateral current spreading effect on the efficiency droop in GaN based light-emitting diodes, J. Disp. Technol. 9 (2013) 266-271.

[19] X.C. Cao, S.D. Arthur, High-power and reliable operation of vertical light-emitting diodes on bulk GaN, Appl. Phys. Let. 85 (2004) $3971-3973$.

[20] H. Kim, S.-N. Lee, Theoretical considerations on current spreading in GaN-based light emitting diodes fabricated with top-emission geometry, J. Electrochem. Soc. 157 (2010) H562-H564.

[21] H.-Y. Ryu, J.-I. Shim, Effect of current spreading on the efficiency droop of InGaN light-emitting diodes, Opt. Express 19 (2011) $2886-2894$.

[22] B. Cao, S. Li, R. Hu, S. Zhou, Y. Sun, Z. Gan, S. Liu, Effect of current crowding on light extraction efficiency of conventional GaN-based light-emitting diodes, Opt. Express 21 (2013) 25381-25388.

[23] O. Li, Y. Li, M. Zhang, W. Ding, F. Yun, Current spreading in GaN-based light-emitting diodes, Chin. Phys. B25 (2016) 117102.

[24] Ü. Özgür, X. Ni, X. Li, J. Lee, S. Liu, S. Okur, V. Avrutin, A. Matulionis, H. Morkoç, Ballistic transport in InGaN-based LEDs: impact on efficiency, Semicons. Sci. Technol. 26 (2011) 014022.

[25] V. Avrutin, S.A. Hafiz, F. Zhang, Ü. Özgür, H. Morkoç, A. Matulionis, InGaN light-emitting diodes: efficiency-limiting processes at high injection, J. Vac Sci. Technol. A 31 (2013) 050809.

[26] V. Sheremet, Metrological aspects of measuring resistance of ohmic contacts, Radioelectron. Commun. Syst. 53 (2010) 119-128.

[27] A.N. Andreev, M.G. Rastegaeva, V.P. Rastegaev, S.A. Reshanov, Current spreading in semiconductors during measurements of the contact resistivity of ohmic contacts, Semiconductors 32 (1998) 739-744.

[28] H.H. Berger, Models for contacts to planar devices, Sol. St. El. 15 (1972) 145-158.

[29] H. Murrmann, D. Wiedmann, Current crowding on metal contacts to planar devices, IEEE Trans. Electr. Dev. 16 (1969) $1022-1024$.

[30] L. Wang, W. Liu, Y. Zhang, Z.-H. Zhang, S.T. Tan, X. Yi, G. Wang, X. Sun, H. Zhu, H.V. Demir, Graphene-based transparent conductive electrodes for GaNbased light emitting diodes: challenges and countermeasures, Nano Energy 12 (2015) 419-436.

[31] J.O. Song, J.-S. Ha, T.-Y. Seong, Ohmic contacts technology for GaN-based light emitting diodes: role of P-Type contact, IEEE Trans. El. Dev. 57 (2010) $42-59$. 